\title{
On Criteria to Choose a Content Management System: A Technology Acceptance Model Approach
}

\author{
Jislane S. S. Menezes ${ }^{1}$, Danilo G. A. Ramos ${ }^{1}$ and Michel S. Soares ${ }^{1}$ \\ ${ }^{1}$ Department of Computing, Federal University of Sergipe, São Cristóvão, Brazil \\ \{jislanesds@gmail.com,dgaramos@gmail.com,mics.soares\}@gmail.com
}

\begin{abstract}
Efficiently choosing software tools is a complex process, as it must consider many variables so that the best software which fulfills the requirements list can be selected. Choosing the correct Content Management System (CMS) for an organization is no different. Many different variables have to be taken into account, and large amount of resources have to be applied. However, mostly the process of choice is based on weak considerations. This paper uses the Technology Acceptance Model (TAM) in order to verify the influence of the evaluation criteria in the process of selecting a CMS. Through a descriptive statistical analysis and the Spearman's correlation coefficient, relations between the three concepts proposed by the TAM model, Perceived Usefulness (PU), Perceived Ease of Use (PEOU) and Perceived Usage (PUE), were evaluated. Data were submitted to non parametric methods and the results confirm two of the three formulated hypothesis: Perceived Ease of Use positively influences Perceived Usage of a CMS (H2) and Perceived Ease of Use positively influences Perceived Usefulness (H3). A case study performed on an IT government company regarding five instances of CMS is proposed in this paper.
\end{abstract}

Index Terms-Technology Acceptance Model, Content Management Systems, Software Acquisition

\section{Introduction}

Information Systems are considered critical success factors for most companies [1]. It is well-known that failures can lead even well-established organizations to

DOI reference number: 10.18293/SEKE2016-092 bankruptcy [2]. With the adoption of information systems by companies and the advent of Internet, organizations are capable of sharing information using web sites and working on projects remotely, connecting their collaborators from around the world. However, organizations have faced many issues for developing and maintaining their web based applications. Their solution is either hiring a technical team to develop and manage the web systems, or hiring a company to perform these tasks. Both solutions have shown to be problematic for reasons such as high costs, slow pace of change, and safety issues. Therefore, Content Management Systems (CMSs) appeared in the mid of the 1990's to facilitate development and management of organizations' web sites [3], as well as managing corporate data and content of organizations on the web.

Selecting the most useful Information System in an efficient way, when too many alternatives do exist, and when multiple criteria are known, is both hard and crucial [4]. Choosing the correct CMS for an organization is no different. Among so many CMS solutions, it is hard for a company to choose the right one after considering the company characteristics' and so many variables, such as cost, ease of use, stability, user comfort, among others, so that the best software which fulfills all the requirements can be selected to the client. In addition, time-pressure creates discomfort and limits the amount of time spent examining available information for decision making [5]. Software acquisition is considered a risky and complex activity [6]. According to results published in [7], risks occurred more frequently in software acquisition than in software development. Decision has also to consider "good enough" solutions, because the high number of criteria and most often the shortage of time makes it difficult to choose the best solution.

This paper creates a theoretical foundation for the development of a model to choose a CMS in order to meet 
customer needs. The model is based on the Technology Acceptance Model - TAM. TAM was initially proposed to evaluate information systems tools [8] [9], but it has also been used in other contexts, such as to shape user attitude toward Mobile Cloud Services [10], to propose a model which explains and predicts the use of software measures [11], among other studies. In our case, we applied TAM to discover most interesting criteria to evaluate CMSs for users. A case study performed on an IT government company regarding five instances of CMS is proposed in this paper.

\section{Background on CMS and TAM}

\section{A. CMS}

A CMS [12] is a software used to develop and maintain websites in an easier way than using a programming language and other software development tools. By using a CMS, a user can create a website, update it, manage the content, post new content without changing the whole code of the page, or having to create new pages from scratch. Everything is managed by the CMS, which may include useful tools such as text editors, multimedia managing, social network integration, layout editors, blog management systems, users management systems and a wide variety of features that may vary from software to software.

There are many available CMSs. A simple search in a common search engine will find more than one hundred instances of CMSs, with many different features and characteristics. CMSs are an alternative to supply the demand of development and maintenance of websites without previous technical knowledge. As the years passed by, CMSs evolved into robust platforms of development that provide a high number of aggregate features, such as text editors, management tools, document management, authorization and workflow, customization and multi lingual publishing. They can be bundled or stand-alone applications and their main goal is to create, manage, deploy and store content on web pages, which can include multimedia files and applications that interact with the user.

\section{B. TAM}

Technology Acceptance Model - TAM [8] is a model created specifically for the study of the usage behavior of computer technologies. TAM model suggests that when users are presented with a new technology, a number of factors influence their decision about how and when they will use it. Originally, TAM incorporates two main variables, Perceived Ease of Use - PEOU, and Perceived Usefulness - PU. A third variable is considered in this research: Perceived Usage - PUE [9].
Perceived Usefulness - PU variable can be defined as "how the person believes that using a certain technology will improve its performance on a certain task" [8]. This is important because given the right idea of how users think the software will help them to achieve their goals on their tasks at work.

Perceived Ease of Use - PEOU variable can be defined as "how the person believes that learning to use a certain technology is without effort" [8]. It is important to know if there will be any resistance from the users to the new technology. Sometimes softwares are very difficult to use, frustrating the users and making their performance to finish their tasks worse than without the software.

Perceived Usage - PUE is the variable that seeks to point out the features that are really used in the software. This variable finds which are the features that have most important use in the tool. Given the right importance to the features, we can see how the software will be used, which features will be used, and the ones not considered by most users.

\section{Methodology}

\section{A. Research Model Proposed}

Since the goal of this work is to understand what criteria influence the attitude of users on the interaction with a CMS, a set of criteria has been grouped in TAM's central variables.

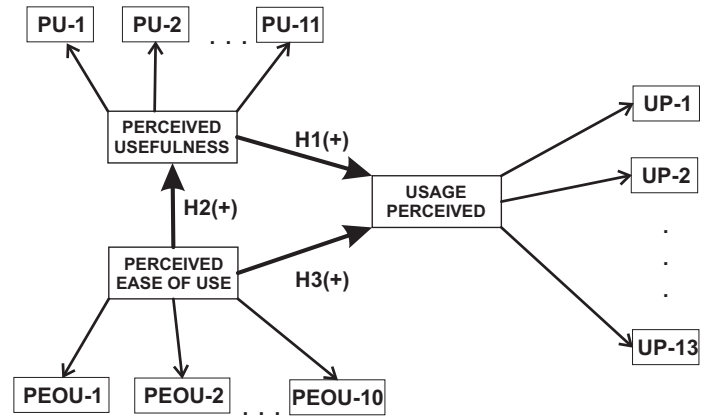

\section{Fig. 1. Research model (adapted from Adams (1992) [9])}

Figure 1 depicts the proposed research model. For the Perceived Usefulness variable, 11 criteria of measurement were set for basic maintenance of a content editor. For the variable Perceived Ease of Use, 10 evaluation criteria were set as learnability, ease of use on the interface, installation and update. For the Perceived Usage variable, 13 criteria were proposed that assess additional features plugins that tend to facilitate frequent interaction between software and user. Given the above model and the need to 
understand how these variables are correlated, we present the following research hypotheses:

H1. Perceived Usefulness positively influences the Perceived Usage to a CMS.

H2. Perceived Ease of Use positively influences Perceived Usage to a CMS.

H3. Perceived Ease of Use positively influences Perceived Usefulness to a CMS.

\section{B. Instrument Development and Validation}

An online questionnaire consisting of items describing the study variables is used as data collection instrument. Design of the questionnaire was informed by literature review, the statement of problems, and research problems. A 5-point Likert scale was proposed to measure perceived attitudes of the employees by providing a range of responses to each statement. The scale ranged from (1) strongly disagree, (2) disagree, (3) neutral, (4) agree, and (5) strongly agree.

The questionnaire, distributed via email to employees of State government departments that interact with some instance of CMS, was designed after interview with experts from an IT Company, a government agency that centralizes development of web portals for all government departments. Selection of criteria noted the items considered important by experts and two user groups were selected: IT Department and Communication Department. Hereby, 20 questionnaires were completed and considered valid for statistical analysis. 34 questions were proposed in the survey, relating to three studied variables, two demographic questions and two questions about the degree of interaction with CMS tools. In the questionnaire, constituent definitions of each variable were described. Presented variables are: Perceived Use (PU - 11), Perceived Easy of Use (PEOU - 10), and Perceived Usage (PUE - 13).

This study employed quantitative methods for analyzing data. Data collected was subjected to a descriptive and inferential analysis involving mean, standard deviation and frequency count. Used technique is the Spearman correlation, since the direction of the relationship was specified in the hypothesis and variables were not normal.

\section{Results}

\section{A. Responses to TAM Questionnaire}

By analyzing averages presented in Tables I, II and III, it turns out that participants of the poll have shown an elevated agreement in relation to most of the items from the survey. This mean they think the applications are very easy to use, are useful and that they used them very often. Besides, from data we can conclude it is common that more than $80 \%$ of users have a positive opinion, (4 or higher) for most of the questionnaire. The positive assessment of respondents showed a $94.6 \%$ participation for PU, a $86.5 \%$ for PEOU and a $75.9 \%$ for PUE. With these results, it is noted that the technology is well received, they hope for software that is easy to use and make their work performance better with basic and additional features. Items that showed a rejection from the poll were PUE9 and PEOU8. Perceived Usefulness variable items showed the higher levels of agreement.

\section{B. CMS Users' Perceived Ease of Use, Usefulness and Use Relations}

In order to check the relations between the CMS users' perceived ease of use, usefulness and use, the Spearman correlation presented the existence of a significant and positive correlation $(\mathrm{Sig}<0.05)$ to relations between PEOU and PU, and between PEOU and PUE. Table IV shows this information more clearly.

Variable Rho values to $\mathrm{H} 2$ and $\mathrm{H} 3$ hypotheses were very close to $\mathrm{H} 1$, which shows a moderate and strong association between variables Perceived Ease of Use and Perceived Usage, and between Perceived Usefulness and Perceived Ease of Use, respectively. Hypothesis H1 has not been confirmed, since it did not achieve less than 0.05 [13] of significance value and Rho's coefficient shows a low correlation. Therefore, even tough users understand that the CMS tool will improve their performance on work activities (PU), this benefit does not relate to the perception of easiness to use the CMS without effort (PEOU). Differently from the other two hypothesis which highlight the easiness of using (PEOU) as an important aspect of a CMS acceptance, as it influences the other variables of this proposal.

Result of hypothesis H3 is corroborated by the study of [14] which confirms the positive influence between Perceived Ease of Use and Perceived Usefulness in more than $75 \%$ of assessed works. No works evaluating the Perceived Usage variable proposed by Adams [9] in relation to other variables were found. With this, hypothesis $\mathrm{H} 2$ and $\mathrm{H} 3$ can be supported.

\section{Case Study}

Five popular Open Source CMS's were chosen to be part of the evaluation process. The evaluation process took ten weeks long, with approximately two weeks per CMS, since the TAM Method requires a certain use and knowledge of the software being analyzed.

For the analysis results, the CMS's are named as CMS1, CMS2, CMS3, CMS4 and CMS5, being CMS1 the first CMS to be evaluated, CMS2 the second one and so on. 
TABLE I. Descriptive statistics PU ( $\mathrm{N}=\mathbf{2 0})$.

\begin{tabular}{|c|c|c|c|c|c|c|c|c|}
\hline Perceived Usefulness - statements 1 to 11 & 1 & 2 & 3 & 4 & 5 & $\mathrm{~m}$ & $s$ & pos \\
\hline 1 - The CMS should allow webpage edition & 0 & 0 & 1 & 9 & 10 & 4.45 & 0.61 & 19 \\
\hline 2 - The CMS should allow update of content that was published previously & 0 & 1 & 0 & 8 & 11 & 4.45 & 0.76 & 20 \\
\hline 3 - The CMS should allow indexing of stored documents & 0 & 0 & 0 & 10 & 10 & 4.5 & 0.51 & 20 \\
\hline 4 - The CMS should allow organization of content by subject & 0 & 0 & 0 & 11 & 9 & 4.45 & 0.51 & 20 \\
\hline 5 - The CMS should allow organization of content via menu & 0 & 0 & 0 & 10 & 10 & 4.5 & 0.51 & 20 \\
\hline 6 - The CMS should provide interactive content containing videos & 0 & 0 & 2 & 8 & 10 & 4.4 & 0.68 & 18 \\
\hline 7 - The CMS should provide interactive content containing audio & 0 & 0 & 3 & 8 & 9 & 4.3 & 0.73 & 17 \\
\hline 8 - The CMS should provide a news area & 0 & 0 & 0 & 6 & 14 & 4.7 & 0.47 & 20 \\
\hline 9 - The CMS should provide layout edition without changing directly source code of the page & 0 & 1 & 4 & 8 & 7 & 4.05 & 0.89 & 16 \\
\hline 10 - The CMS should provide a text edition tool & 0 & 0 & 0 & 10 & 10 & 4.5 & 0.51 & 20 \\
\hline 11 - The CMS should be compatible with most web browsers. & 0 & 0 & 0 & 5 & 15 & 4.75 & 0.44 & 20 \\
\hline
\end{tabular}

TABLE II. Descriptive statistics PEOU ( $N=20)$.

\begin{tabular}{|l|c|c|c|c|c|c|c|c|}
\hline Perceived ease of use of CMS - statements 1 to 10 & 1 & 2 & 3 & 4 & 5 & $\mathrm{~m}$ & $\mathrm{~s}$ & $\mathrm{pos}$ \\
\hline 1 - The CMS should be easy to learn & 0 & 0 & 1 & 8 & 11 & 4.5 & 0.61 & 19 \\
\hline 2 - The CMS should be easy to install & 0 & 0 & 2 & 9 & 9 & 4.35 & 0.67 & 18 \\
\hline 3 - The CMS should be easy to install in various operational systems & 0 & 0 & 3 & 9 & 8 & 4.25 & 0.72 & 17 \\
\hline 4 - The CMS should be easy to update & 0 & 0 & 0 & 6 & 14 & 4.7 & 0.47 & 20 \\
\hline 5 - For me, using a CMS is often frustrating & 0 & 8 & 6 & 4 & 2 & 3 & 1.02 & 14 \\
\hline 6 - The CMS should be flexible to interact & 0 & 1 & 0 & 14 & 5 & 4.15 & 0.67 & 20 \\
\hline 7 - The CMS should facilitate memory on how to make tasks & 0 & 0 & 3 & 12 & 5 & 4.1 & 0.64 & 17 \\
\hline 8 - The CMS should have a self explanatory interface & 0 & 0 & 3 & 10 & 7 & 4.2 & 0.7 & 17 \\
\hline 9 - The CMS should facilitate insertion of images on a text & 0 & 0 & 0 & 10 & 10 & 4.5 & 0.51 & 20 \\
\hline 10 - The CMS should facilitate text diagramming & 0 & 0 & 0 & 8 & 12 & 4.6 & 0.5 & 20 \\
\hline
\end{tabular}

TABLE III. Descriptive statistics PUE (N = 20).

\begin{tabular}{|c|c|c|c|c|c|c|c|c|}
\hline Perceived Usage - statements 1 to 13 & 1 & 2 & 3 & 4 & 5 & $\mathrm{~m}$ & s & pos \\
\hline 1 - The use of file sharing methods on the CMS increases my performance at work & 0 & 0 & 6 & 8 & 6 & 4 & 0.8 & 14 \\
\hline $\begin{array}{l}2 \text { - The notification by e-mail in interactions in } \\
\text { new posts increases my performance at work }\end{array}$ & 0 & 2 & 5 & 9 & 4 & 3.75 & 0.91 & 15 \\
\hline 3 - The comment approval feature increases my performance at work & 0 & 2 & 6 & 8 & 4 & 3.7 & 0.92 & 14 \\
\hline 4 - The reply comments feature increases my performance at work & 0 & 0 & 6 & 9 & 5 & 3.95 & 0.76 & 14 \\
\hline $\begin{array}{l}5 \text { - The classification of documents stored on the } \\
\text { system increases my performance at work }\end{array}$ & 0 & 0 & 4 & 11 & 5 & 4.05 & 0.69 & 16 \\
\hline 6 - The use of content search tools combining categories and keywords makes my work easy & 0 & 0 & 1 & 10 & 9 & 4.4 & 0.6 & 19 \\
\hline 7 - The discussion forums feature increases my performance at work & 0 & 1 & 8 & 9 & 2 & 3.6 & 0.75 & 12 \\
\hline 8 - The poll creation feature increases my performance at work & 0 & 0 & 5 & 9 & 6 & 4.05 & 0.76 & 15 \\
\hline 9 - The blog creation feature increases my performance at work & 1 & 0 & 11 & 5 & 3 & 3.45 & 0.95 & 9 \\
\hline 10 - The remote access for content edition increases my performance at work & 1 & 0 & 1 & 10 & 8 & 4.2 & 0.95 & 19 \\
\hline 11 - The RSS feeds feature makes my work easier. & 0 & 0 & 5 & 11 & 4 & 3.95 & 0.69 & 15 \\
\hline $\begin{array}{l}12 \text { - The integration with social networks (Facebook, } \\
\text { Twitter) increases my performance at work }\end{array}$ & 0 & 1 & 1 & 7 & 11 & 4.4 & 0.82 & 19 \\
\hline 13 - The link incorporated to text creation makes my work easier & 0 & 0 & 1 & 7 & 12 & 4.55 & 0.61 & 19 \\
\hline
\end{tabular}

TABLE IV. Correlation between hypothesis and Spearman coefficient

\begin{tabular}{|c|c|c|}
\hline Hypothesis & Rho Coefficient & Sig. \\
\hline H1 - Perceived usefulness positively influences perceived usage to a CMS & .389 & .090 \\
\hline H2 - Perceived ease-of-use positively influences perceived usage to a CMS. & .468 & .038 \\
\hline H3 - Perceived ease-of-use positively influences perceived usefulness to a CMS. & .681 & .001 \\
\hline
\end{tabular}

Each CMS was analyzed by two evaluators. The two evaluators analyzed the CMS's independently and evaluated them via an online form. When the analysis was finished, each evaluator filled the form based on their own thoughts about the CMS. The form had the questions previously shown and explained in the TAM section of this paper, and the answers were in a 3 point scale.

In order to create the test environment on PHP based
CMS's, Wampserver 2.5 was used as ambient. Wampserver is an application which installs a web development ambient with Apache 2.4.9, PHP 5.5.12 and MySQL 5.6.17, it also comes with PHPMyAdmin as a tool to facilitate database management. On the Python based CMS the tools were a Vagrant kit with a VirtualBox virtual machine and the Putty SSH to simulate a web server to run the CMS. Tables V, VI and VII represents the results of the CMSs evaluation using 
TABLE V. Resulting Table PU

\begin{tabular}{|l|c|c|c|c|c|c|}
\hline PU & CMS1 & CMS2 & CMS3 & CMS4 & CMS5 & Weight \\
\hline 1 & 1 & 1 & 0.5 & 1 & 1 & 4.45 \\
\hline 2 & 1 & 0.75 & 1 & 1 & 1 & 4.45 \\
\hline 3 & 1 & 1 & 0.75 & 0.75 & 1 & 4.5 \\
\hline 4 & 1 & 1 & 0.75 & 1 & 1 & 4.45 \\
\hline 5 & 1 & 1 & 1 & 1 & 1 & 4.5 \\
\hline 6 & 1 & 1 & 1 & 0.75 & 1 & 4.4 \\
\hline 7 & 0.75 & 1 & 0.75 & 0.75 & 0.75 & 4.3 \\
\hline 8 & 1 & 0.5 & 0.5 & 1 & 1 & 4.7 \\
\hline 9 & 1 & 0.25 & 0 & 0.75 & 0.25 & 4.5 \\
\hline 10 & 1 & 1 & 0.5 & 1 & 1 & 4.05 \\
\hline 11 & 1 & 1 & 1 & 1 & 1 & 4.5 \\
\hline \hline
\end{tabular}

TABLE VI. Resulting Table PEOU

\begin{tabular}{|l|c|c|c|c|c|c|}
\hline PEOU & CMS1 & CMS2 & CMS3 & CMS4 & CMS5 & Weight \\
\hline 1 & 1 & 0.25 & 0 & 0.75 & 1 & 4.5 \\
\hline 2 & 1 & 0.75 & 0.5 & 0.25 & 0.25 & 4.35 \\
\hline 3 & 1 & 0.75 & 0.75 & 0.5 & 0.25 & 4.25 \\
\hline 4 & 0.75 & 0.5 & 0.75 & 0.5 & 0.5 & 4.7 \\
\hline 5 & 1 & 0.5 & 0 & 0.5 & 1 & 3 \\
\hline 6 & 1 & 0.5 & 0.25 & 1 & 0.75 & 4.15 \\
\hline 7 & 1 & 0 & 0 & 1 & 1 & 4.1 \\
\hline 8 & 1 & 0.25 & 0.25 & 1 & & 4.2 \\
\hline 9 & 1 & 1 & 1 & 1 & 1 & 4.5 \\
\hline 10 & 1 & 0.5 & 0.5 & 1 & 1 & 4.6 \\
\hline \hline
\end{tabular}

TABLE VII. Resulting Table PUE

\begin{tabular}{|l|c|c|c|c|c|c|}
\hline PUE & CMS1 & CMS2 & CMS3 & CMS4 & CMS5 & Weight \\
\hline 1 & 1 & 1 & 0.75 & 1 & 1 & 4 \\
\hline 2 & 0.5 & 0.25 & 0.25 & 0.25 & 0.5 & 3.75 \\
\hline 3 & 1 & 0 & 0.5 & 0.5 & 1 & 3.7 \\
\hline 4 & 1 & 0.25 & 1 & 1 & 1 & 3.95 \\
\hline 5 & 0.75 & 1 & 0.75 & 0.75 & 1 & 4.05 \\
\hline 6 & 0.75 & 0.75 & 0.5 & 1 & 0.75 & 4.4 \\
\hline 7 & 0.5 & 0.5 & 1 & 0.5 & 0.5 & 3.6 \\
\hline 8 & 0.5 & 0.5 & 0.25 & 0.5 & 0.25 & 4.05 \\
\hline 9 & 1 & 0.5 & 0.5 & 0.75 & 0.5 & 3.45 \\
\hline 10 & 0.5 & 0.5 & 0.5 & 0.75 & 0.75 & 4.2 \\
\hline 11 & 1 & 1 & 1 & 1 & 1 & 3.95 \\
\hline 12 & 1 & 0.75 & 0.5 & 0.5 & 0.75 & 4.4 \\
\hline 13 & 1 & 1 & 1 & 1 & 1 & 4.55 \\
\hline \hline
\end{tabular}

the validated TAM criteria by the interviewed subjects on the Section Results of this paper. Each mean will be a weight in order to evaluate the CMSs.

Each item was evaluated receiving value $0,0.5$ or 1 . As each CMS was evaluated by two evaluators, the final value was the mean between the evaluations. These tables serve as a guideline to help stakeholders to define which CMS is better considering their purposes and requirements. In order to calculate the final scores for each CMS, each evaluated value was multiplied by the mean found by TAM-based questionnaire, presented in Section Results of this paper. Fig. 2 depicts the final scores for each CMS, for each TAM variable.

Following the graphic of Perceived Usefulness (PU), the evaluated softwares answer above $70 \%$ of the items featured in Table 3, highlighting the CMS1 with $97.8 \%$ of agreement in relation to the other CMSs. In relation to Perceived Ease of Use, CMS1 got $97.2 \%$ in the total evaluation. Hence, this CMS shows advantages in items such as Ease of Use and deployment, which decreases the learning curve. CMS3 did not get the best results, specially for the difficulty to find manuals and tutorials available for research, besides being non-intuitive in most operations. As for Perceived Usage, the CMSs attended $81 \%, 63 \%$ and $65.5 \%$ respectively. This result shows that the softwares attended a reasonable quantity of features considered important by the interviewees, as for instance, document classification, the incorporation of hyperlinks in the text and social networks integration.

\section{Threats to Validity}

This study has endeavored to establish a research model for the successful evaluation of CMS systems at an IT government company. However, the study has some limitations that can be addressed in future research. The study had 20 people surveyed, which is a relatively low number for a reliable estimate. However, the survey was answered by subjects working at various state organizations and departments. The method presented in this paper can be replicated with more respondents by other researchers. In terms of scope, this study is confined to the State government departments. Future research could include more public and private institutions that are using CMS.

The response categories in Likert scale has rank order, but the intervals between values cannot be presumed equal [15]. As a result, Likert scales fall within the ordinal level of measurement. When treated as ordinal data, Likert responses can be analyzed using non-parametric tests, such as the Mann-Whitney test, the Wilcoxon signed rank test, and the Kruskal-Wallis test [16]. Non-parametric statistical methods were used to analyze the findings because the subjects involved in the study were few and not chosen randomly from a large population. Objective here was to find representative power of results, and not necessarily statistical significance. It is worthwhile to note that nonparametric techniques do not solve the problem of potential dependency between the answers of participants.

\section{Conclusion}

The process of choosing a software application is a critical factor observed by companies. Large amount of time and financial resources have to be applied for software acquisition. Mostly, the process of choice is based in weak considerations. Considering that, this research aimed to establish evaluations criteria based on TAM, which is an 


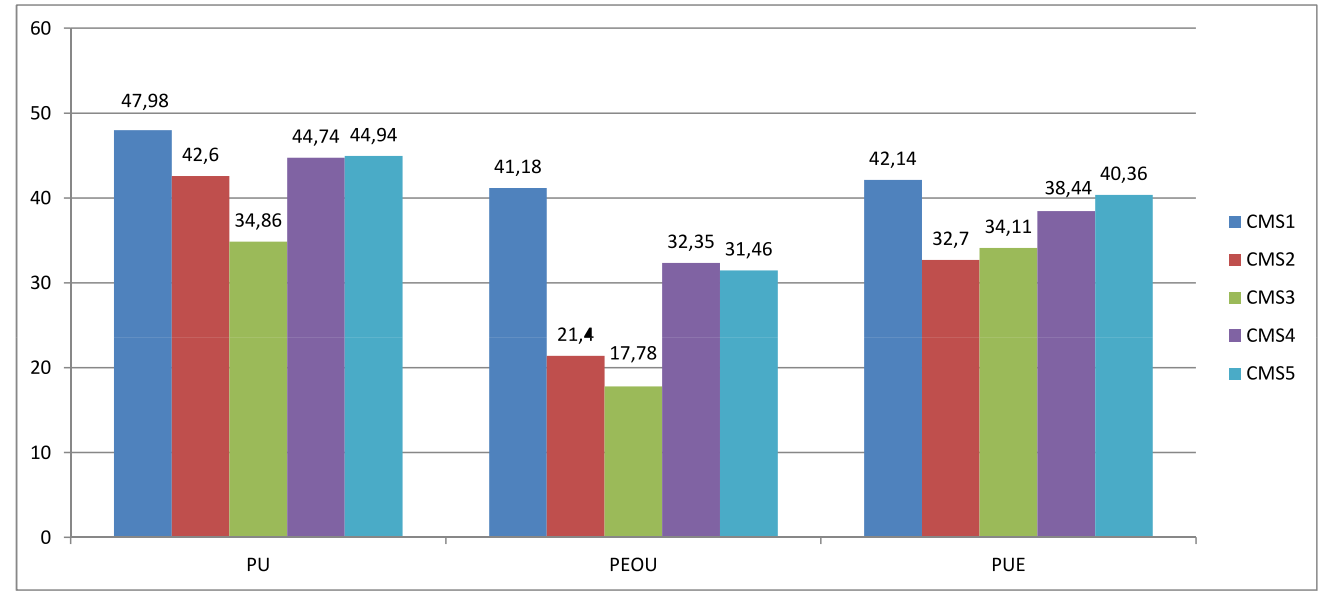

Fig. 2. Final results.

empirical evaluation method applied to comprehend use and behaviour from users of a certain technology.

The research had as purpose to verify the influence of CMS tools evaluation criteria on the relationship between acceptance and use of those systems. It can be seen in this research that users who interact with CMS systems are stimulated to use the product due to the Perceived Ease of Use, the Perceived Usefullness and the Perceived Usage. Whereas there were no rejections from the analyzed variables, it was also observed that the variable Perceived Usage is influenced by variable Perceived Ease of Use. These findings allow to identify what criteria should be considered during a successful evaluation process to choose a CMS tool. For future research, the relation between some variables and user profile can be analyzed.

\section{Acknowledgment}

The authors would like to thank research agency FAPITEC/SE (grant 9614), Governo do Estado de Sergipe, and NAP-CPD EMGETIS (http://www.emgetis.se.gov.br).

\section{References}

[1] S. Petter, W. H. DeLone, and E. R. McLean, "Information Systems Success: The Quest for the Independent Variables," J. of Management Information Systems, vol. 29, no. 4, pp. 7-62, 2013

[2] Y. Dwivedi, D. Wastell, S. Laumer, H. Henriksen, M. Myers, D. Bunker, A. Elbanna, M. Ravishankar, and S. Srivastava, "Research on Information Systems Failures and Successes: Status Update and Future Directions," Information Systems Frontiers, pp. $1-15,2014$

[3] M. White, The Content Management Handbook, 1st ed. Facet Publishing, 2005.

[4] F. Daneshgar, G. C. Low, and L. Worasinchai, "An Investigation of Build vs. Buy Decision for Software Acquisition by Small to Medium Enterprises," Information and Software Technology, vol. 55, no. 10, pp. 1741-1750, 2013.
[5] D. Fehrenbacher and S. Smith, "Behavioural Affect and Cognitive Effects of Time-pressure and Justification Requirement in Software Acquisition: Evidence from an Eye-Tracking Experiment," in 20th Americas Conference on Information Systems, 2014.

[6] S. Harnisch, "Enterprise-level Packaged Software Acquisition: a Structured Literature Review Through the Lens of IT Governance," in European Conf. on Information Systems, April 2014.

[7] D. Kusumo, M. Staples, L. Zhu, H. Zhang, and R. Jeffery, "Risks of Off-the-Shelf-Based Software Acquisition and Development: A Systematic Mapping Study and a Survey," in 16th Int. Conf. on Evaluation Assessment in Software Engineering, May 2012, pp. 233-242.

[8] F. D. Davis, "Perceived Usefulness, Perceived Ease of Use, and User Acceptance of Information Technology," MIS Quarterly, vol. 13, no. 3, pp. 319-339, 1989.

[9] D. A. Adams, R. R. Nelson, and P. A. Todd, "Perceived Usefulness, Ease of Use, and Usage of Information Technology: a Replication," MIS Quarterly, vol. 16, no. 2, pp. 227-247, 1992.

[10] E. Park and K. J. Kim, "An Integrated Adoption Model of Mobile Cloud Services: Exploration of Key Determinants and Extension of Technology Acceptance Model," Telemat. Inf., vol. 31, no. 3, pp. 376-385, 2014

[11] L. G. Wallace and S. D. Sheetz, "The Adoption of Software Measures: A Technology Acceptance Model (TAM) Perspective," Information Management, vol. 51, no. 2, pp. 249-259, Mar. 2014.

[12] S. Baxter and L. Vogt, "Content Management System," 2002, uS Patent 6,356,903. [Online]. Available: https://www.google.com/patents/US6356903

[13] A. Field, Discovering Statistics using SPSS. Sage publications, 2009.

[14] P. Legris, J. Ingham, and P. Collerette, "Why do People Use Information Technology? A Critical Review of the Technology Acceptance Model," Information \& management, vol. 40, no. 3, pp. 191-204, 2003.

[15] S. Jamieson, "Likert Scales: How to (ab)use them," Med. Educ., vol. 38, no. 12, pp. 1217-1218, 2004.

[16] G. W. Corder and D. I. Foreman, Nonparametric Statistics for NonStatisticians: A Step-by-Step Approach. Hoboken, NJ, USA: John Wiley \& Sons, 2009. 